\title{
Oxygen $2 s$ spectroscopy of tin oxides with synchrotron radiation-induced photoemission
}

\author{
J.-M. Themlin, J.-M. Gilles* and R.L. Johnson** \\ Groupe de Physique des Etats Condensés, URA 783 du CNRS, Faculté des Sciences de Luminy, Case \\ 901, 13288 Marseille cedex 9, France \\ * LASMOS, Facultés Universitaires Notre-Dame de la Paix, 61 rue de Bruxelles, 5000 Namur, Belgium \\ ** II Institute for Experimental Physics, Uni. Hamburg, 149 Luruper Chaussee, 2000 Hamburg 52, \\ Germany
}

\begin{abstract}
Oxygen $2 s$ spectroscopy can be especially useful in studies using synchrotron radiation (SR) where the deeper $\mathrm{O} 1 s$ level frequently can not be excited. For tin oxides ( $\mathrm{SnO}$ and $\mathrm{SnO}_{2}$ ), the weak emission from the $\mathrm{O} 2 s$ levels has been previously found degenerate with the intense photoemission peak from $\mathrm{Sn} 4 d$ levels around a binding energy (BE) of $26 \mathrm{eV}$. By working at the Cooper minimum of the interfering tin signal, a distinct peak near $B E \approx 22.5 \mathrm{eV}$ could be unambiguously attributed to emission from $\mathrm{O} 2 s$ levels in tin oxides considering photoionization cross-section arguments. The $\mathrm{O} 2 s$ intensity could now be used for the quantitative evaluation of the near-surface oxygen-species concentration. We have used the O $2 s$ in combination with the $\mathrm{Sn} 4 d$ areas to study the variations of the [Sn]/[O] ratio as a function of the preparation of a $\mathrm{SnO}_{2}$ single crystal. Although the dominant formal valence state for tin determined from a $\mathrm{Sn} 4 d$ lineshape analysis remains essentially $\mathrm{Sn}^{4+}$, we show that this $[\mathrm{Sn}] /[\mathrm{O}]$ ratio can vary from simple to double. This can be explained by oxygen interstitials buried in a sub-surface region during ion bombardment, which segregate at the surface upon annealing.
\end{abstract}

\section{INTRODUCTION}

In detailed photoemission studies, we have recently addressed the electronic structure of tin oxides ( $\mathrm{SnO}$ and $\mathrm{SnO}_{2}$ ) using UPS [1] and XPS [2]. The low-energy region ( $0 \mathrm{eV}<$ Binding Energies (BE) $<35 \mathrm{eV}$ ) has proved particularly useful for discriminating between stannous and stannic oxide. Using XPS, the deconvolution of the $\mathrm{Sn} 3 d$ peak in terms of $\mathrm{Sn}^{2+}$ and $\mathrm{Sn}^{4+}$ formal valence states can be usefully guided [2] by considering characteristic features of the VB (which derives from $\mathrm{O} 2 p, \mathrm{Sn} 5 s$ and $5 p$ levels ), e.g. the energy difference between the VB leading peak and the shallow $\mathrm{Sn} 4 d$ levels found at $\mathrm{BE} \approx 26 \mathrm{eV}$. With UPS which allows better resolving power, the direct deconvolution of these Sn $4 d$ levels is usually possible [3]. Oxygen $2 s$ spectroscopy [4] can be especially useful in studies using synchrotron radiation (SR) where the deeper O $1 s$ level frequently can not be excited. However, other $s$ or $p$ levels often hybridize with $\mathrm{O} 2 s$ levels while the high intensity from more localized $d$ or $f$ levels may also hide the low-intensity $\mathrm{O} 2 s$ emission, found between binding energies of 14 and $33 \mathrm{eV}$. This is dramatically true for systems involving oxygen and tin, since the $O 2 s$ levels have been reported to be degenerate [5] with the intense $\mathrm{Sn} 4 d$ signal, strongly favoured by its electron occupancy ( 10 electrons vs 2 electrons for $\mathrm{O} 2 s$ ) and its partial photoelectron cross section [6] $\sigma_{\mathrm{Sn} 4 d}\left(\sigma_{\mathrm{Sn} 4 d}>10 \sigma_{\mathrm{O} 2 s}\right.$ in most of the X-ray and UV regimes ). Nevertheless, both in our UPS and monochromatized-XPS spectra, we have noticed on the low-BE side of the $\mathrm{Sn} 4 d$ peak a very small bump at $\mathrm{BE} \approx 22 \mathrm{eV}$. In order to identify this unknown structure, which, we think, could be due to $\mathrm{O} 2 s$ levels, we have tried to reduce the intensity of the interfering tin signal. This has been possible by working at the Cooper minimum of the Sn $4 d$ levels. This minimum, which occurs for states with nodes in their radial wave function [7], has been calculated [6] for $\mathrm{Sn}$ at about $140 \mathrm{eV}$. 

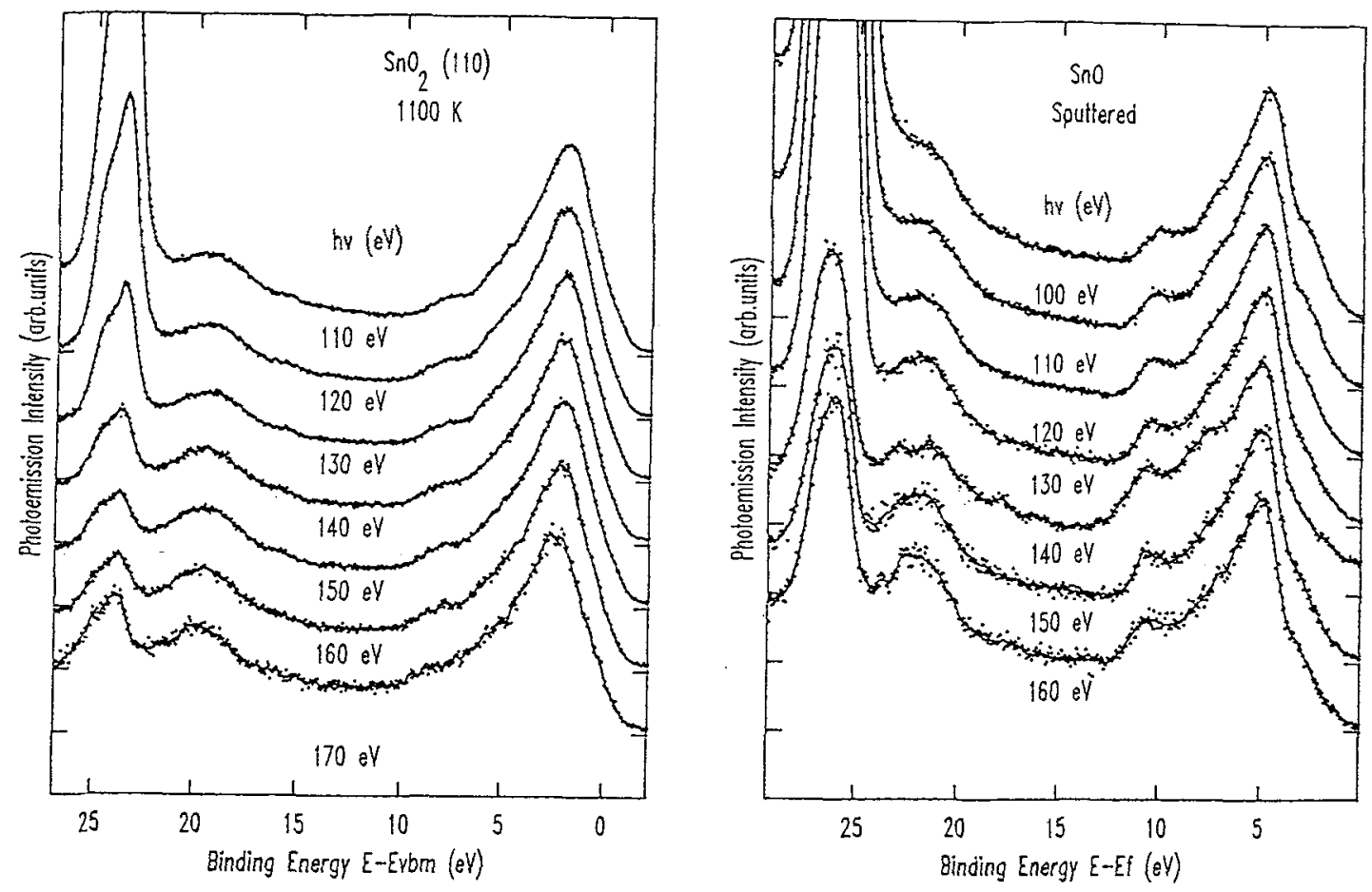

Fig.1: VB and near-core region EDC's for photon energies in the vicinity of the Cooper minimum of the $\mathrm{Sn} 4 d$ levels, for $\mathrm{SnO}_{2}$ (110) annealed at $1100 \mathrm{~K}$ (left panel ) and sputtered $\mathrm{SnO}$ ( right panel).

\section{RESULTS}

Fig. 1 shows representative UPS spectra of $\mathrm{SnO}_{2}$ and $\mathrm{SnO}$ taken at various photon energies in the vicinity of the Cooper minimum. The overall degradation of the signal-to-noise ratio for increasing $h v$ reflects the general decrease of the partial $\sigma_{i}$. To compensate for this overall decrease and to ease comparison, each spectrum has been normalized to the intensity of the prominent peak of the VB, derived from levels which have mainly an $\mathrm{O} 2 p$ character [8], and is easily located in the VB of both oxides. For tin dioxide (Fig.1, left panel), the Sn $4 d$ intensity rapidly decreases with hv up to $150 \mathrm{eV}$ where it appears as intense as the unknown structure. The same trend is reproduced for tin monoxide, except that the $\mathrm{Sn} 4 d$ area does not decrease below $\approx 3$ times the unknown structure area. As a general trend, it is visible that the unknown structure intensity slightly increases with respect to the $02 p$ intensity between 110 and $170 \mathrm{eV}$.

\section{INTERPRETATION}

The hypothesis of a satellite associated with $\mathrm{Sn} 4 d$ levels can directly be ruled out since the unknown-structure intensity does not follow the large variations of the $\mathrm{Sn} 4 d$ peak intensity. Assuming that the unknown structure could be due to emission from the $\mathrm{O} 2 s$ levels, we have plotted in Fig. 2 the ratio of its intensity and the $\mathrm{O} 2 p$ intensity (extracted from the VB ). These points correlate rather well with the theoretical ratio of the partial photoionization cross sections $\sigma_{\mathrm{O}} 2 s / \sigma_{\mathrm{O} 2 p}$ between 110 and $170 \mathrm{eV}$. The same type of argument has been previously used to identify an $\mathrm{O} 2 \mathrm{~s}$-derived stucture in the VB photoemission spectra of high-temperature superconductors [9]. We therefore unambiguously attribute the weak emission at $\mathrm{BE} \approx 22.5 \mathrm{eV}$ in tin oxides to $\mathrm{O} 2 \mathrm{~s}$ levels. This energy lies in the range where $\mathrm{O} 2 s$ levels have been reported for other oxides, e.g. from $20.3 \mathrm{eV}$ for $\mathrm{BaO}[10]$ to 24.5 for $\mathrm{SiO}_{2}$ [4]. 


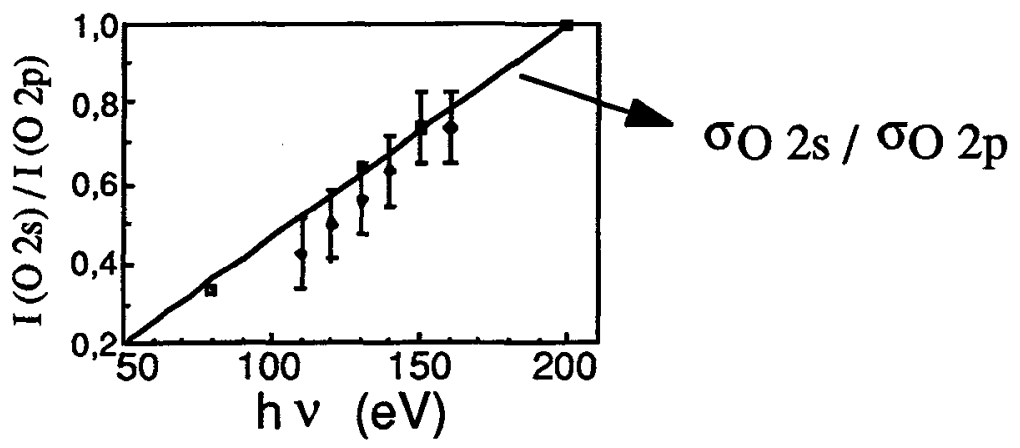

Fig.2: Correlation between the intensity ratio ( solid points ) of the unknown structure ( supposedly $\mathrm{O} 2 s$-derived) and the $\mathrm{O} 2 p$ structure in the $\mathrm{VB}$ of $\mathrm{SnO}_{2}(110)$ with the calculated partial photoionization cross sections ratio $\sigma_{\mathrm{O} 2 s} / \sigma_{\mathrm{O} 2 p}$ between 110 and $170 \mathrm{eV}$ ( solid line ).

We have now a direct access to an oxygen core level intensity and we can use the $\mathrm{O} 2 \mathrm{~s}$ intensity or its area to compare it with the $\mathrm{Sn} 4 d$ intensity, without relying on the hybridized $\mathrm{O} 2 p$ orbitals which are much more difficult to isolate in the valence band of the oxides. This allows the determination of the relative concentrations of oxygen and metal within the photoelectron sampling depth. It also cancels a drawback of UPS applied to oxides, which, considering the low photon energies implied, does not usually allow electrons from the $\mathrm{O} 1 s$ to be excited above the vacuum level. For example, one can now understand qualitatively why the intensity ratio I ( $\mathrm{Sn} 4 d)$ / I $(\mathrm{O} 2 s)$ is higher for tin monoxide ( 3.5 for $\mathrm{SnO}$ vs 1.5 for $\mathrm{SnO}_{2}$ ), since it simply reflects the stoichiometry of the oxide. In what follows, we will illustrate the usefulness of our ability to estimate the [Sn]/[O] ratio through the $\mathrm{O} 2 s$ and $\mathrm{Sn} 4 d$ area measured at the Cooper minimum.

\section{APPLICATION}

We have measured in this way the I ( $\mathrm{Sn} \mathrm{4d)} / \mathrm{I}(\mathrm{O} 2 s)$ intensity ratio for a fresh $\mathrm{SnO}_{2}(110)$ single crystal face prepared in the usual way using successive Ar-ion bombardment and UHV annealing treatments. Although the Sn $4 d$ levels and the VB show only minor changes [3], Fig.3 denotes substantial changes of the [Sn]/[O] ratio, and particularly, an irreversible decrease of the post-sputtering ratio with the number of sputtering/annealing cycles. This appears rather surprising if one considers the usually reported trends for most oxides which are easily reduced [11] by preferential sputtering of the light oxygen atoms under Ar-ion bombardment. Such a trend is of course locally valid here since ion bombardment effectively increases the $[\mathrm{Sn}] /[\mathrm{O}]$ ratio, which increases further with a larger ion dose (treatments $n^{\circ} 4$ and 5 ). This is also confirmed by the analysis of the $\mathrm{Sn} 4 d$ detailed peak shape and by the VB which show evidence of some limited reduction (evolution towards an SnO stoechiometry) upon ion bombardment [3]. Annealing, on the contrary, restores near-core and VB spectra characteristic of stoichiometric $\mathrm{SnO}_{2}$, which is reflected by the (local) decrease of the [Sn]/[O] ratio.

In the present case, we believe that the net $[\mathrm{Sn}] /[\mathrm{O}]$ decrease may be explained by the creation, during ion bombardment, of a subsurface layer enriched in oxygen. Part of this oxygen diffuses towards the surface upon annealing, causing the progressive decrease of the [Sn]/[O] ratio. We describe this mechanism in more detail hereafter. The dominant defect in $\mathrm{SnO}_{2}$, the doubly ionized oxygen vacancy, has been determined using electrical methods [12]. We propose, therefore, that the oxygen species detected here are neutral atomic oxygens, which are virtually undetectable in a conductibility experiment. The creation of such an "anti-frenkel" disorder [13] where both oxygen vacancies and oxygen interstitials coexist is likely to happen upon Ar-ion bombardment. Considering momentum conservation arguments, the oxygen primaries hit by $\mathrm{Ar}^{+}$ions are displaced towards the bulk, resulting in the formation of a subsurface layer enriched in neutral atomic oxygen interstitials. The latter remain undetected in UPS due to the short sampling depth. Upon annealing, these oxygen species could well diffuse towards the surface through the microdomains reduced by the ion bombardment [3], and even along the c-axis of the native rutile structure of $\mathrm{SnO}_{2}$ (diffusion parallel to the surface). As another proof of the oxygen diffusion phenomena, we have also checked that the absolute intensity of the $\mathrm{O} 2 \mathrm{~s}$ emission increases during annealing, while the Sn $4 d$ absolute intensity remains the same. 


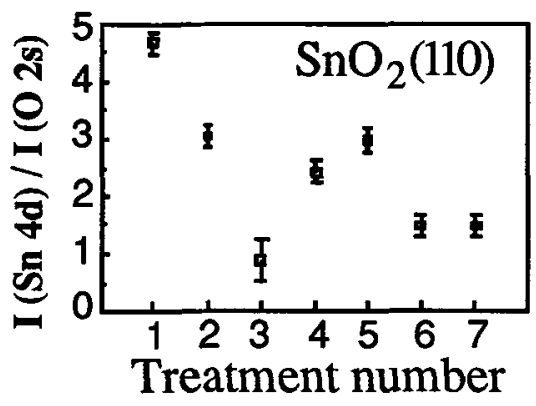

\author{
$n^{\circ 1}$ : Ar- ion Bombardment ( 122 a.u. ) \\ $\mathrm{n}^{\circ} 2$ : Annealing 1000K \\ $\mathrm{n}^{\circ} 3$ : Annealing 1050K \\ $n^{\circ} 4$ : Ar- ion Bombardment ( 65 a.u.) \\ $\mathrm{n}^{\circ} 5$ : Ar -ion Bombardment ( 185 a.u.) \\ $\mathrm{n}^{\circ} 6$ : Annealing $1000 \mathrm{~K}$ \\ $n^{\circ} 7$ : Ar-ion and annealing $1100 \mathrm{~K}$
}

\begin{abstract}
Fig.3: I (Sn 4d) / I (O 2s) area ratio measured at the Cooper minimum for seven successive treatments of a freshly-introduced $\mathrm{SnO}_{2}$ single crystal exposing its (110) face. The arbitrary units indexing each ion bombardment are the product of the incident current times the duration of the ion beam exposure. An evolution towards low $[\mathrm{Sn}] /[\mathrm{O}]$ ratios is visible.
\end{abstract}

In conclusion, we want to emphasize the distinction between the oxide composition based on the relative concentrations of cations in formal valence state $\mathrm{Sn}^{4+}$ or $\mathrm{Sn}^{2+}$, determined through lineshape analysis of the $\mathrm{Sn} 4 d$ levels, and oxide composition based on the [Sn]/[O] ratio obtained through the I (Sn 4d) / I (O 2s) area ratio measured at the Cooper minimum. Although the relative concentrations of $\mathrm{Sn}^{4+}$ (dominant species ) and $\mathrm{Sn}^{2+}$ cations take reproducible values characteristic of sputtering or annealing [3], Fig.3 reveals a two-fold variation of the 1 ( $\mathrm{Sn} 4 d) / \mathrm{I}(\mathrm{O} 2 s)$ intensity ratio after some cleaning cycles. Such large differences could explain why former Auger studies [14] mainly based on [Sn]/[O] ratios often showed large variations or irreversible changes with sample history. It is thus clear that the relative "stability" of UPS spectra which are usually exploited through their "chemical bonding" or valence state informations rather than through the atomic concentrations point-of-view could well keep important phenomena hidden. The determination of these atomic concentrations requires the availability of an oxygen core level with an area comparable with the chosen metal core line. We have shown that for tin oxides, $\mathrm{O} 2 s$ levels are ideal candidates for that purpose. $\mathrm{O} 2 s$ spectroscopy using SRinduced photoemission is thus useful to study diffusion processes in tin oxides and can bring valuable information to the study of oxygen/tin systems.

Acknowledgments: This research was supported by the belgian "Fonds National de la Recherche Scientifique" and by the "Actions de Recherches Concertées" of the Belgian Ministry for Science Policy.

\title{
References:
}

[1] J.-M. Themlin, R. Sporken, J. Darville, R. Caudano, J.-M. Gilles, R.L. Johnson, Phys. Rev. B 42 (1990) 11914-11925

[2] J.-M. Themlin, M. Chtaïb, L. Henrard, P. Lambin, J. Darville and J.-M. Gilles, Phys. Rev. B 46 (1992) 2460-2466

[3] J.-M. Themlin, Ph.D. thesis, Facultés Universitaires Notre-Dame de la Paix, Namur (1991), unpublished

[4] W. Ranke and H.J. Kuhr, Phys. Rev.B 39 (1989) 1595

[5] P.A. Cox, R.G. Egdell, C. Harding, W.R. Patterson and P.J. Tavener, Surf. Sci.123 (1982) 179

[6] J.J. Yeh and I. Lindau, Atomic data and nuclear data tables 32 (1985) 1-155

[7] G. Margaritondo and A. Franciosi, Ann. Rev. Mater. Sci. 14 (1984) 67-93

[8] S. Munnix and M. Schmeits, Phys. Rev. B 27 (1983) $7624 ; 33$ (1986) 4136

[9] M. Tang et al, Phys. Rev. B 38 (1988) 897

[10] K. Jacobi, C. Astaldi, B. Frick, and P. Geng, Phys. Rev. B 36 (1987) 3079

[11] V.E. Henrich, Rep. Prog. Phys. 48 (1985) 1481

[12] S. Samson and C.G. Fonstad, J. Appl. Phys. 44, 4618 (1973)

[13] H.J. Matzke, Nonstoichiometric Oxides (Materials Science and Technology Series, ed. O.T. Sorensen, Academic Press, New-York, 1981) p.161

[14] E. de Frésart, J. Darville and J.-M. Gilles, Surf. Sci. 126 (1983) 518 